\title{
O Impacto da Orientação para o Mercado e da Orientação para Aprendizagem sobre a Inovação de Produto: uma Comparação entre a Indústria Eletroeletrônica e o Setor de Ensino Universitário de Administração
}

\author{
Marcelo Gattermann Perin \\ Cláudio Hoffmann Sampaio \\ Sandro Nero Faleiro
}

\begin{abstract}
Resumo
Apesar da consolidação teórica do tema Orientação para o Mercado e sua influência no desempenho organizacional, uma corrente de autores da área de Marketing tem argumentado que a Orientação para o Mercado é necessária mas não suficiente para sustentar uma vantagem competitiva de longo prazo. Visando ao aprofundamento deste tema, este artigo tem como objetivo principal investigar a relação entre Orientação para o Mercado, Orientação para Aprendizagem e Inovação. Para tanto, um modelo teórico, contendo as relações hipotetizadas entre os referidos construtos, foi desenvolvido e testado, por meio da aplicação da técnica de modelagem de equações estruturais. Os resultados do levantamento realizado na Indústria Eletro-Eletrônica do Brasil e nos Cursos de Graduação em Administração filiados à ANGRAD (Associação Nacional dos Cursos de Graduação em Administração) demonstraram uma influência positiva e significativa da Orientação para o Mercado sobre a Inovação de produtos, além do impacto indireto da Orientação para Aprendizagem na Inovação mediante a sua forte e positiva influência sobre a Orientação para o Mercado. Resultados complementares, futuras pesquisas e implicações gerenciais foram, ainda, discutidos.
\end{abstract}

Palavras-chaves: orientação para o mercado; orientação para aprendizagem; inovação.

\begin{abstract}
In spite of the theoretical consolidation of the theme Market Orientation and its influence on the organizational performance, a group of authors in the Marketing area has been arguing that the Market Orientation is necessary but not enough to sustain a competitive advantage for a long period. Trying to study more in depth this theme, this article presents the results of a research developed to investigate the relationship among Market Orientation, Learning Orientation and Innovation. For this purpose, a theoretical model, containing the hypothetical relationships among the referred constructs, was developed and tested through the application of the structural equations modeling methodology. The results of two surveys, developed in the Electro-Electronic Industry of Brazil and in the undergraduate courses in Business Administration associated to ANGRAD (Associação Nacional dos Cursos de Graduação em Administração), have demonstrated a positive and significant influence of the Market Orientation on the Innovation of Products, besides the indirect impact of the Learning Orientation on the Innovation through its strong and positive influence on Market Orientation. Complemental results, future researches and managerial implications were, also, discussed.
\end{abstract}

Key words: market orientation; learning orientation; innovation. 


\section{INTRODUÇÃO}

A Orientação para o Mercado recebeu substancial interesse acadêmico e de profissionais de Marketing ao longo dos últimos anos (Becker e Homburg, 1999), especialmente na sua relação com o construto de desempenho organizacional (Uncles, 2000), tendo gerado um número significativo de trabalhos e claro avanço na teoria sobre o tema (Day, 1994b; Narver, Slater e Tietje, 1998). Muitos autores da área de Marketing, contudo, têm concordado em que a Orientação para o Mercado é necessária, mas não suficiente para sustentar uma vantagem competitiva de longo prazo (Slater e Narver, 1995; Dickson, 1996; Baker e Sinkula, 1999a; Farrell, 2000). A posição desta corrente de autores é a de que a habilidade de se engajar em processos de aprendizagem organizacional e de inovação deve acompanhar a Orientação para o Mercado, para que as organizações alcancem a vantagem competitiva de longo prazo, particularmente em ambientes dinâmicos e competitivos.

Aprofundando os estudos sobre esses temas, uma série de trabalhos empíricos buscou demonstrar a relevância da aplicação dos conceitos de Orientação para o Mercado e orientação para a aprendizagem, individual e associadamente, para o sucesso da empresa e da inovação (Slater e Narver, 1995; Claycomb e Germain,1997; Sinkula, Baker e Noordewier, 1997; Han et. al., 1998; Hurley e Hult, 1998; Baker e Sinkula, 1999a, 1999b; Farrell, 2000; Lukas e Ferrell, 2000; Slater e Narver, 2000), como forma de as organizações manterem e sustentarem vantagens em seus mercados.

Contudo, a despeito do grande número de publicações específicas sobre os temas de Orientação para o Mercado e Orientação para Aprendizagem (individualmente considerados), ressalta-se a afirmação de Baker e Sinkula (1999a) sobre a abundância de proposições teóricas e a escassez de comprovações empíricas da relação indireta da Orientação para Aprendizagem sobre o desempenho empresarial (e sobre a Inovação de Produtos), por meio da potenciação da Orientação para o Mercado, especialmente na efetivação da aprendizagem, e toma-se como considerável o espaço ainda existente para a investigação da relação entre estes construtos e deles com a Inovação (Slater e Narver, 1995; Baker e Sinkula, 1999a).

Neste sentido, o presente artigo apresenta os detalhes de um estudo ${ }^{(1)}$ que objetivou investigar empiricamente a relação entre Orientação para o Mercado, Orientação para Aprendizagem e Inovação. O estudo restringiu-se a dois setores 
de atividade - Indústria Eletroeletrônica e Cursos de Graduação em Administração filiados à Associação Nacional dos Cursos de Graduação em Administração (ANGRAD), buscando comparar os resultados de setores com ênfase e contexto notadamente distintos. Os resultados encontrados trazem relevantes implicações acadêmicas e gerenciais, contribuindo para a ampliação dos trabalhos que abordam esses temas como modelos integrados.

\section{Referencial Teórico}

O referencial teórico descrito a seguir aborda os construtos de Orientação para o Mercado, Orientação para Aprendizagem e Inovação, além dos estudos que relacionam os referidos construtos.

\section{Orientação para o Mercado}

A partir do início da década de 1990, com os trabalhos seminais de Kohli e Jaworski (1990,1993) e de Narver e Slater (1990 e 1994), o tema Orientação para o Mercado, e sua relação com o desempenho, tem ocupado o centro da teoria e prática da administração de marketing (Day, 1994b; Becker e Homburg, 1999). Ao longo da última década, consolidou-se uma teoria dos antecedentes e conseqüências da Orientação para o Mercado, desenvolveram-se medidas para o construto e testou-se o impacto da Orientação para o Mercado sobre o desempenho empresarial. Apesar da existência de outras linhas importantes de pesquisa (Deshpandé, Farley e Webster, 1993; Day, 1994b; Deshpandé e Farley, 1998), os trabalhos de Kohli e Jaworski $(1990,1993)$ e de Narver e Slater (1990, 1994) serão analisados como referenciais para o estudo de Orientação para o Mercado, devido ao destaque a eles oferecido pela literatura da área (Perin, 2002).

Narver e Slater (1990) delinearam a Orientação para o Mercado como um construto unidimensional constituído por três componentes comportamentais (orientação para o cliente, orientação para os concorrentes e coordenação interfuncional) que seriam as ações e os padrões de comportamento da empresa, investigando a relação da Orientação para o Mercado com o desempenho empresarial. Os autores constataram também que existe pouca influência dos moderadores ambientais sobre a relação entre Orientação para o Mercado e desempenho empresarial, pois os benefícios apresentados pela Orientação para o Mercado são de longo prazo, enquanto as condições ambientais são passageiras (Slater e Narver, 1994). 
A pesquisa realizada por Kohli e Jaworski (1990), buscando estruturar o construto de Orientação para o Mercado, apoiou a Orientação para o Mercado em três pontos básicos, quais sejam, a geração de inteligência de mercado, a disseminação desta inteligência de mercado e a resposta da empresa à inteligência gerada e disseminada. A validade empírica destas proposições foi testada por Jaworski e Kohli (1993), com a formulação de um modelo para mensurar o grau de Orientação para o Mercado de uma empresa, baseado na proposição dos antecedentes e das conseqüências da Orientação para o Mercado. Tendo por base os seus estudos anteriores (Kohli e Jaworski, 1990; Jaworski e Kohli, 1993), Kohli, Jaworski e Kumar (1993) desenvolveram a escala MARKOR, com o intuito de servir como instrumento para medir o grau de Orientação para o Mercado das empresas.

A partir do delineamento do construto de Orientação para o Mercado e de escalas para sua mensuração, a verificação empírica da relação entre Orientação para o Mercado e desempenho tem sido foco central de diversos estudos, conduzidos em diferentes indústrias, setores e contextos culturais. De maneira geral, observa-se uma relação positiva entre Orientação para o Mercado e desempenho empresarial (Sampaio, 2000; Perin, 2002).

\section{Orientação para Aprendizagem}

A Orientação para Aprendizagem é tida como um conjunto de "valores e práticas que refletem onde a aprendizagem ocorre e a natureza do que foi aprendido” (Nevis, Dibella e Gould, 1995, p. 76). Para Baker e Sinkula (1999a, p. 413), a Orientação para Aprendizagem é "o conjunto de valores que influencia o grau com que a organização está satisfeita com as suas teorias em uso, modelos mentais e a lógica dominante”, isto é, um conjunto de valores organizacionais que influenciam a propensão da organização para criar e utilizar o conhecimento. Assim, empresas com alto grau de Orientação para Aprendizagem estimulam nos seus funcionários, ou mesmo exigem deles, o questionamento constante das normas da organização que orientam o seu processamento de informações de mercado e as ações organizacionais.

A aprendizagem organizacional tem sido investigada no nível de aprendizagem de processo, ou orientação para a aprendizagem, e envolve a aprendizagem de ciclo simples (ou adaptativa), na qual a empresa reage aos eventos do ambiente; a aprendizagem de ciclo duplo (ou generativa), que compreende a modificação da visão de mundo da empresa e conseqüente descarte de procedimentos e sistemas obsoletos; e a aprendizagem deutero que é definida por Probst e Buchel (1997) como a habilidade de aprender a aprender, consistindo no ganho de insights sobre o processo de aprendizagem. 
Sinkula, Baker e Noordewier (1997) relacionam três valores organizacionais normalmente associados à predisposição da empresa em aprender: compromisso com a aprendizagem, postura de mente aberta e visão compartilhada.

Para estes autores, empresas que têm compromisso com a aprendizagem valorizam a necessidade de entender as causas e os efeitos de suas ações, permitindo-lhes a detecção e correção de erros na teoria em uso. A característica de mente aberta está associada ao conceito de unlearning, através do qual a empresa proativamente questiona até mesmo as suas mais antigas rotinas, asserções e crenças, descartando ou substituindo o conhecimento ultrapassado. A visão compartilhada, por sua vez, difere do compromisso com a aprendizagem e da postura de mente aberta, na medida em que influencia a direção da aprendizagem, enquanto os outros dois valores influenciam a intensidade da aprendizagem (Sinkula, Baker e Noordewier, 1997). O compartilhamento dos propósitos e objetivos da organização entre os seus membros propicia o foco para a aprendizagem, promovendo esforço, compromisso e propósito entre os membros da organização (Day, 1994a).

Para Baker e Sinkula (1999a) a Orientação para Aprendizagem afeta diretamente a habilidade de desafiar as asserções ou as verdades antigas sobre o mercado e de como a empresa deve ser organizada para tratá-las, promovendo a inovação baseada em novos paradigmas e impulsionando a organização para além da hábil resposta às mudanças do ambiente, refletindo o valor que a organização dá ao desafio das asserções que modelam o seu relacionamento com o ambiente (Baker e Sinkula, 1999a).

\section{Inovação}

Entre os requisitos que aparecem como fundamentais para que as empresas obtenham sucesso competitivo em seus mercados, aparece a necessidade de não só reagir com sucesso às demandas mas também de influenciar o ambiente no qual elas se inserem (Slater e Narver, 1995). Acreditando nessa afirmação, há muito tempo tem-se direcionado relevância à Inovação (Hurley e Hult, 1998). Drucker (1954) já destacava essa importância, ressaltando ser a Inovação uma das funções essenciais para a empresa atingir seu objetivo superior de satisfação dos clientes.

Apesar da não recentidade dos estudos sobre Inovação, existe ainda hoje uma dificuldade em defini-la, uma vez que uma Inovação pode dar-se em relação ao produto, ao processo tecnológico, à estrutura administrativa ou ao programa relacionado com os membros da organização (Kimberly, 1981). Tushman e Nadler (1997) definem dois tipos básicos de Inovação: Inovação de Produto e Inovação de Processo. 
Especificamente neste estudo, a ênfase será dada à Inovação de Produtos, que é definida como o processo de trazer ao uso do mercado novos produtos e/ou novas tecnologias (Lukas e Ferrell, 2000). Nesta mesma linha, Galbraith (1997) associa Inovação à geração de um produto resultante do desenvolvimento de nova idéia. Já Tushman e Nadler (1997) definem Inovação como a criação de um produto que seja novo para a unidade de negócios da empresa, enfatizando que a maioria das inovações são resultados de mudanças incrementais de produtos.

Os estudos de Baker e Sinkula (1999b) e de Han, Kim e Srivastava (1998) apontam para a Inovação de produtos como um elemento chave do sucesso sustentável e da performance empresarial, decorrendo em aumento de interesse de estudos relacionados a explorar os antecedentes da Inovação (Hurley e Hult, 1998), assim como barreiras e facilitadores ao processo de Inovação (Henard e Szymanski, 2001).

Montoya-Weiss e Calantone (1994) realizaram extensa revisão de literatura, identificando 18 fatores como precedentes da Inovação. Numa mesma linha de pesquisa, Henard e Szymanski (2001) realizaram uma metanálise sobre os principais estudos que abordavam a Inovação e identificaram 24 antecedentes, apontando vantagem de produto, potencial de mercado, conhecimento das necessidades do cliente, competência nas tarefas de pré-desenvolvimento e recursos dedicados à Inovação como os mais significativos antecedentes sobre o desempenho de novos produtos.

Damanpour (1991) e Tushman e Nadler (1997) procuraram identificar variáveis organizacionais que teriam impacto positivo e negativo sobre a Inovação. De maneira geral, os diferentes estudos apresentaram resultados similares. Como principais barreiras à Inovação aparecem a aversão ao risco, as recompensas baseadas em padrões tradicionais, a burocracia, a cultura e estrutura conservadora da empresa, as rivalidades internas, as hierarquias empresariais complexas, rígidas e centralizadas. De forma inversa, a coordenação e relação interfuncional, a capacidade de incentivar e recompensar novas idéias, a atitude gerencial positiva em relação à mudança e a cultura mais voltada para o mercado aparecem como principais facilitadores.

\section{Relação entre OM, OA e Inovação}

A literatura de Marketing tem tratado a Orientação para Aprendizagem como intimamente ligada ao construto de Orientação para o Mercado (Day, 1994a; Sinkula, 1994; Slater e Narver, 1995; Dickson, 1996; Sinkula, Baker e Noordewier, 1997; Hurley e Hult, 1998; Baker e Sinkula, 1999a). Do ponto de vista da Orientação para o Mercado, o arcabouço teórico da aprendizagem organizacional 
é valioso para a organização, uma vez que estimula o foco no entendimento e satisfação das necessidades dos clientes, não apenas as expressas mas também as latentes, por meio de novos produtos e serviços, bem como novas formas de atuar no negócio (Day, 1994b; Sinkula, 1994; Slater e Narver, 1995; Dickson, 1996). Esta condição leva a organização a melhores resultados, tais como sucesso na inovação (Slater e Narver, 1995; Hurley e Hult, 1998).

Embora teoricamente enfatizem uma relação entre os construtos investigados - Orientação para o Mercado, Orientação para Aprendizagem e Inovação, muitos autores da área de Marketing afirmam que poucos estudos empíricos buscaram comprovar essa relação (Sinkula e Baker; Noordwier, 1997; Claycomb e Germain, 1997; Baker e Sinkula, 1999a). Em termos gerais, os referidos poucos estudos verificaram que a Orientação para Aprendizagem afeta indiretamente o desempenho organizacional, pelo aumento dos comportamentos orientados para o mercado, e afeta diretamente o desempenho, facilitando a aprendizagem generativa que leva à Inovação de Produtos, procedimentos e sistemas (Slater e Narver, 1995; Dickson, 1996; Claycomb e Germain, 1997; Hurley e Hult, 1998; Baker e Sinkula, 1999a; Slater e Narver, 2000).

Dentre os estudos que abordam os construtos de forma integrada, destacam-se os trabalhos de Baker e Sinkula (1999a, 1999b). Baker e Sinkula (1999b), declaradamente influenciados pelos trabalhos de Han, Kim e Srivastava (1998) e de Hurley e Hult (1998), inseriram o construto de Inovação no modelo testado pelo seu estudo anterior (Baker e Sinkula, 1999a). Na verdade, Baker e Sinkula (1999b) passaram a considerar o sucesso de novos produtos, que antes era tratado como dimensão do desempenho organizacional, como sendo novo construto (Inovação), antecedente do desempenho organizacional (Figura 1).

\section{Figura 1: Relação entre Orientação para o Mercado, Orientação para Aprendizagem, Inovação e Desempenho Organizacional}

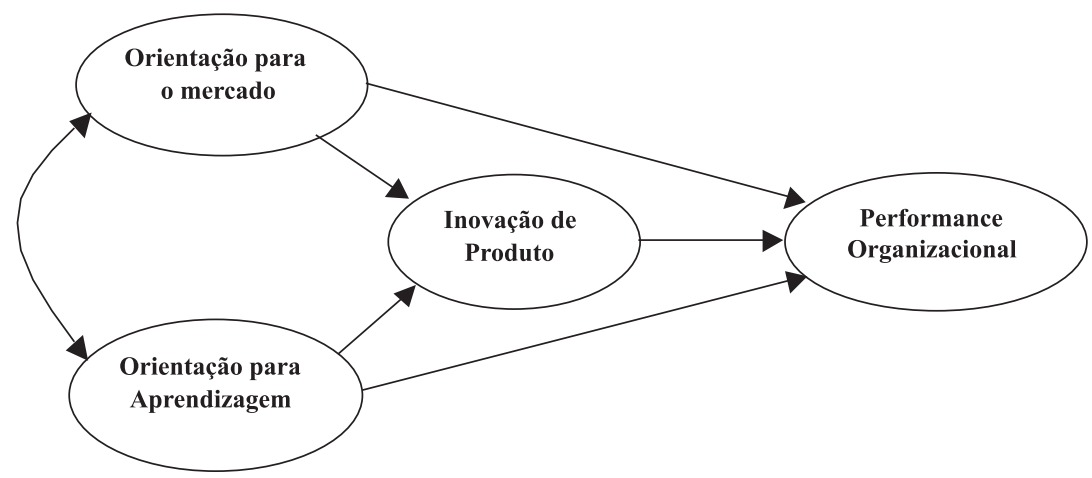

Fonte: Baker e Sinkula (1999b, p. 299). 
As hipóteses configuradas no novo modelo teórico, apresentado na Figura 1, foram testadas e comprovadas no estudo em exame. Assim, na discussão sobre os resultados de seu estudo, Baker e Sinkula (1999b, p.14) afirmam que "a Orientação para o Mercado e a Orientação para Aprendizagem são a chave para o sucesso do desempenho dirigido pela Inovação”, isto é, a Orientação para o Mercado e a Orientação para Aprendizagem influenciam o desempenho empresarial por meio de seus efeitos diretos sobre a Inovação. A conclusão mais controversa, no entanto, foi a de inexistência de efeito direto da Orientação para o Mercado sobre o desempenho organizacional, diferentemente dos resultados da sua pesquisa anterior (Baker e Sinkula, 1999a). Ressalta-se que a diferença básica entre os dois estudos foi o fato de Baker e Sinkula (1999b) considerarem o sucesso de novos produtos (ou Inovação) como construto antecedente do desempenho e não uma dimensão do desempenho organizacional, conforme Baker e Sinkula (1999a).

O modelo de Baker e Sinkula (1999b), apresentado na Figura 1, foi tomado como base para o modelo teórico testado no presente estudo. Esta escolha ocorreu em função da relativa estabilidade que o modelo teórico proposto por Baker e Sinkula (1999b) vem recebendo na literatura específica de Marketing, além da solidez metodológica registrada no processo de seu desenvolvimento (Sinkula, 1994; Sinkula, Baker e Noordewier, 1997; Baker e Sinkula, 1999a; Baker e Sinkula, 1999b). O modelo testado, no entanto, não incluiu o construto de desempenho organizacional, restringindo-se às demais relações constantes do modelo de Baker e Sinkula (1999b). Os procedimentos metodológicos aplicados nesta pesquisa para a avaliação do modelo proposto são descritos a seguir.

\section{Método}

O método aplicado na realização desta pesquisa, dada a sua natureza descritiva, foi o levantamento do tipo survey cross-sectional, seguindo recomendações de Churchill (1999) e Malhotra (2001). A técnica utilizada para a avaliação do modelo proposto foi a análise fatorial confirmatória, com a aplicação de modelagem de equações estruturais (Hoyle, 1995; Hair et al., 1998). Para tanto, tomou-se como base os dados coletados em dois levantamentos do tipo survey, respectivamente nos setores de Cursos de Graduação em Administração e da Indústria Eletroeletrônica do Brasil.

Conforme postulado por Hoyle (1995) e Hair et al. (1998), a aplicação da 
análise fatorial confirmatória foi precedida de uma avaliação da correlação entre os construtos do modelo para identificação de possíveis relações não incluídas no modelo, ou ainda a fragilidade das relações teoricamente hipotetizadas.

Os detalhes da elaboração dos instrumentos de coleta aplicados, do processo de coleta de dados realizado e dos procedimentos de análise de dados são apresentados a seguir.

\section{Elaboração do Instrumento de Coleta}

A avaliação do modelo teórico proposto exigiu a elaboração de instrumento de coleta para a mensuração dos construtos de Orientação para o Mercado, de Orientação para Aprendizagem e de Inovação.

A validação de conteúdo do instrumento de coleta foi efetuada pelo método de juízes, acadêmicos e práticos (Malhotra, 2001). Os juízes, dois acadêmicos com experiência na aplicação dos conceitos da área de Marketing, consideraram as escalas apropriadas. Após essa etapa, para avaliação dos termos empregados, em ambos os campos de estudo, os questionários foram apresentados a quatro executivos de cada ramo em análise com experiência na área, escolhidos por julgamento do pesquisador, objetivando aproximar os termos empregados nos respectivos instrumentos de coleta a uma linguagem mais prática e mais específica dos setores em estudo. Alguns termos foram adaptados de acordo com as sugestões apresentadas.

Complementarmente, os instrumentos de coleta de dados foram pré-testados em um conjunto de oito empresas de cada população-alvo, representando diferentes portes e localizações geográficas, com o objetivo de identificar e eliminar problemas potenciais de entendimento e preenchimento. O pré-teste indicou homogeneidade de compreensão pelas organizações consultadas.

Por indicação de Hair et al. (1998) e Garver e Mentzer (1999), a avaliação de ambos os instrumentos foi realizada por meio do cálculo de confiabilidade e de variância extraída a partir da soma das cargas das variâncias padronizadas e dos erros de mensuração das variáveis. Todos os constructos em análise apresentaram consistência interna (Hair et al., 1998).

Para operacionalização do construto de Orientação para o Mercado foi utilizada a escala MARKOR (Kohli, Jaworski e Kumar, 1993) por apresentar testes de confiabilidade e validade aceitos em países e culturas distintos dos EUA (Pitt et al., 1996). Para o levantamento na Indústria Eletroeletrônica, a 
Orientação para o Mercado foi mensurada por meio das três dimensões originais da escala MARKOR - geração de inteligência de mercado, disseminação de inteligência de mercado e ação de resposta, sendo composta por um conjunto total de vinte indicadores (Kohli, Jaworski e Kumar, 1993), com escala Likert de 5 pontos. Utilizou-se, para tanto, a tradução da escala MARKOR para a língua portuguesa apresentada por Perin (2002). Já no levantamento realizado nos Cursos de Graduação em Administração, dada as peculiaridades do ambiente de ensino superior, foram consideradas as adaptações feitas por Caruana, Ramaseshan e Ewing (1996) sobre a escala MARKOR. Estes autores utilizaram a MARKOR para investigar o efeito da Orientação para o Mercado sobre o desempenho de universidades australianas e neozelandesas, realizando algumas adaptações na MARKOR em função das características particulares do segmento pesquisado. Utilizou-se, para tanto, a tradução da escala MARKOR, adaptada para o ambiente acadêmico, apresentada por Faleiro (2001), também com Likert de 5 pontos.

A Orientação para Aprendizagem foi operacionalizada a partir da escala proposta por Sinkula, Baker e Noordewier (1997) e revisada por Baker e Sinkula (1999a). A escolha desta escala foi devida ao seu estágio de confiabilidade e validação. Vários estudos já aplicaram e validaram esta escala para a mensuração da Orientação para Aprendizagem, entre eles: Sinkula, Baker e Noordewier (1997), Claycomb e Germain (1997), Baker e Sinkula (1999a), Baker e Sinkula (1999b) e Farrell (2000). Para Sinkula, Baker e Noordewier (1997), a Orientação para Aprendizagem é composta por três dimensões: comprometimento com a aprendizagem, a visão compartilhada e a mente aberta, cada qual com seus indicadores específicos. Para a mensuração de cada um dos indicadores do construto de Orientação para Aprendizagem, respeitou-se a configuração original proposta por Baker e Sinkula (1999a), mediante a aplicação de escala do tipo Likert de 5 pontos. Utilizou-se, neste caso, a tradução da escala apresentada por Perin (2002).

Para mensurar o grau de Inovação dos Cursos de Graduação em Administração foi adotado o constructo sugerido por Baker e Sinkula (1999b). Segundo estes autores, o construto de Inovação é formado por quatro variáveis relacionadas ao pioneirismo no mercado, ao grau de diferenciação dos novos produtos, à taxa de introdução de novos produtos e ao grau de sucesso dos novos produtos. No caso da Indústria Eletroeletrônica, os indicadores foram definidos, seguindose o padrão empregado nos estudos realizados por Claycomb e Germain (1997), Hurley e Hult (1998), Baker e Sinkula (1999b) e Slater e Narver (2000). Os três indicadores utilizados mensuram a Inovação, considerando a intensidade, pioneirismo e sucesso de novos produtos. A mensuração de cada um dos seus indicadores, por sua vez, foi subjetiva e realizada por meio de escala de 5 pontos (Churchill, 1999), variando de 1 (Muito MENOR que o dos principais 
concorrentes) a 5 (Muito MAIOR que o dos principais concorrentes), seguindo as recomendações identificadas na revisão da literatura da área e respeitando a familiaridade dos respondentes com a escala de cinco pontos.

\section{Coleta de Dados}

O survey aplicado no Setor de Ensino de Graduação em Administração envolveu os cursos filiados à Associação Nacional dos Cursos de Graduação em Administração - ANGRAD. Com isto, a população foi constituída por 346 cursos de Administração distribuídos por todo o Brasil. Já o survey aplicado à Indústria Eletroeletrônica considerou a população de empresas deste setor afiliadas à Associação Brasileira da Indústria Elétrica e Eletrônica (ABINEE), sendo composta por um total de 541 organizações.

A forma de coleta aplicada para os Cursos de Graduação em Administração foi o correio eletrônico, enquanto para a Indústria Eletroeletrônica, utilizou-se o questionário via correio convencional, tendo sido ambos enviados para todos os membros das respectivas populações. O destino dos questionários foi para a alta administração das empresas envolvidas (no caso das empresas afiliadas à ABINEE) ou para o diretor de Escola ou Faculdade, chefe de departamento ou coordenador do curso de Administração (no caso dos cursos afiliados à ANGRAD).

Destaca-se, portanto, que não existiu processo de amostragem para o envio dos instrumentos de pesquisa (Malhotra, 2001). As amostras efetivamente utilizadas para as análises dos dados foram formadas pelos questionários devolvidos e considerados válidos em cada um dos setores. Assim, já excluídos os casos de endereços errados, de erros de preenchimento ou de questionários incompletos, a amostra na Indústria Eletroeletrônica foi composta por 179 casos e no setor de Cursos de Graduação em Administração por 123 casos. Em ambos os levantamentos, portanto, obteve-se uma taxa de resposta efetiva superior a $33 \%$.

As amostras, contudo, não podem ser consideradas como probabilísticas, uma vez que a probabilidade de inclusão de cada membro da população na amostra não é conhecida e tampouco equivalente (Churchill, 1999; Malhotra, 2001). De toda forma, com o intuito de amenizar os efeitos da amostragem não probabilística, e também em função do método de coleta utilizado questionário via correio (eletrônico ou convencional), realizou-se nas duas amostras a análise do viés dos não-respondentes mediante teste de comparação entre ondas ( $1^{\mathrm{a}}$ onda após o envio do questionário e $2^{\mathrm{a}}$ onda em seguida à pós-notificação) e da comparação entre as características das 
empresas respondentes e não respondentes (dados relativos ao número de funcionários, localização geográfica e classificação pelo código de atividade). Os resultados obtidos pelos dois procedimentos indicaram a inexistência de viés nos dados colhidos.

Os dados de entrada foram ainda submetidos a uma análise de normalidade uni e multivariada, considerando o cálculo dos valores de skewness e kurtosis, além da medida de Mardia de kurtosis multivariada (West, Finch e Curran, 1995; Hoyle, 1995; Hair et al., 1998; Ullman, 2000). Esta análise registrou a ocorrência de não normalidade em algumas das variáveis manifestas do modelo. Ainda que o nível da não normalidade diagnosticada tenha sido considerado baixo, este fato exigiu cuidado especial na seleção do método de estimação a ser aplicado para a avaliação das equações estruturais, bem como na utilização das medidas de ajustamento (Chou e Bentler, 1995) ${ }^{(2)}$.

\section{Método de Estimação e Medidas de Ajustamento}

No presente estudo, seguiu-se a recomendação explícita oferecida pela empresa SmallWaters Corp., produtora do software estatístico AMOS ${ }^{\circledR}$ utilizado na pesquisa. Em sua homepage (http://www.smallwaters.com/amos/ faq), dado o diagnóstico de não normalidade "moderada" (skewness menor ou igual a dois e kurtosis menor ou igual a sete), o tamanho de amostra inferior a 200 casos e o uso de variáveis categóricas do tipo Likert com cinco ou mais pontos, a empresa sugere que seja aplicada a própria abordagem maximum likelihood (ML), considerando a variável categórica como intervalar e aplicando o processo de estimação de bootstrapping ${ }^{(3)}$ para a correção dos erros padronizados. Destaca-se que esta abordagem é corroborada por West, Finch e Curran (1995).

Já os indicadores de estimação utilizados para a validação do modelo foram selecionados do conjunto proposto por Chou e Bentler (1995) e por Hair et al. (1998). Os indicadores considerados têm sido freqüentemente empregados em artigos que utilizam modelagem de equações estruturais para avaliar os construtos de Orientação para o Mercado e Orientação para Aprendizagem, em especial aqueles que empregaram escalas categóricas do tipo Likert e com registro de não normalidade dos dados (Fritz, 1996; Pelham, 1997; Egeren e O’Connor, 1998; Siguaw, Simpson e Baker, 1998; Baker e Sinkula, 1999a; Becker e Homburg, 1999; Farrell, 2000; Sampaio, 2000; Perin, 2002).

Destaca-se que há várias medidas de ajustamento desenvolvidas, absolutas e comparativas, não existindo, portanto, um único coeficiente que resuma o ajustamento do modelo. Além disso, não existem parâmetros rígidos para essas 
medidas para aceitação ou rejeição de um modelo, dependendo de fatores como a complexidade do modelo proposto (Hair et al., 1998).

As medidas absolutas de ajustamento aplicadas, que determinam o grau em que o modelo geral prediz a matriz de covariância ou de correlação observada (Hair et al., 1998), foram: Qui-quadrado sobre graus de liberdade (?2 / GL), Goodness-of-fit (GFI) e Root Mean Square Error of Approximation (RMSEA). Já as medidas comparativas de ajustamento, que comparam o modelo proposto ao modelo nulo (null model), foram: Adjusted Goodness-of-fit (AGFI), TuckerLewis Index (TLI) e Comparative Fit Index (CFI). De acordo com Hair et al. (1988), considera-se como aceitável o ajustamento de um modelo quando o $\div 2$ / GL apresenta valor igual ou inferior a 5; GFI, AGFI, TLI e CFI apresentam valores iguais ou superiores a 0,8 aceitaveis; e o RMSEA apresenta valores entre 0,05 e 0,08 .

\section{Modelo de Mensuração}

A análise fatorial confirmatória seguiu os passos recomendados por Hair et. al. (1998). O modelo analisado considerou a Orientação para o Mercado e a Orientação para Aprendizagem como construtos de segunda ordem (Bagozzi e Edwards, 1998), compostos por dimensões específicas. Assim, a Orientação para o Mercado foi definida pelas dimensões de geração de inteligência, disseminação de inteligência e resposta ao mercado, conforme a proposição básica da escala MARKOR. A Orientação para Aprendizagem foi modelada pelas dimensões de compromisso com a aprendizagem, visão compartilhada e postura de mente aberta, respeitando a proposição inicial do modelo de Baker e Sinkula (1999a). O construto de Inovação, por sua vez, foi modelado como sendo de primeira ordem composto pelos indicadores supracitados.

A estimação e a avaliação das medidas de ajustamento, contudo, exigiram a simplificação do modelo para a obtenção de parcimônia na estimação dos parâmetros, dado o tamanho da amostra e a complexidade do modelo de mensuração (considerado o número de variáveis manifestas e latentes). O relativo consenso na literatura aponta a necessidade de ao menos cinco casos observados por parâmetro a ser estimado (Hair et al., 1998). A Figura 2 apresenta o modelo de mensuração utilizado. 


\section{Figura 2: Modelo de Mensuração da Análise Fatorial Confirmatória}

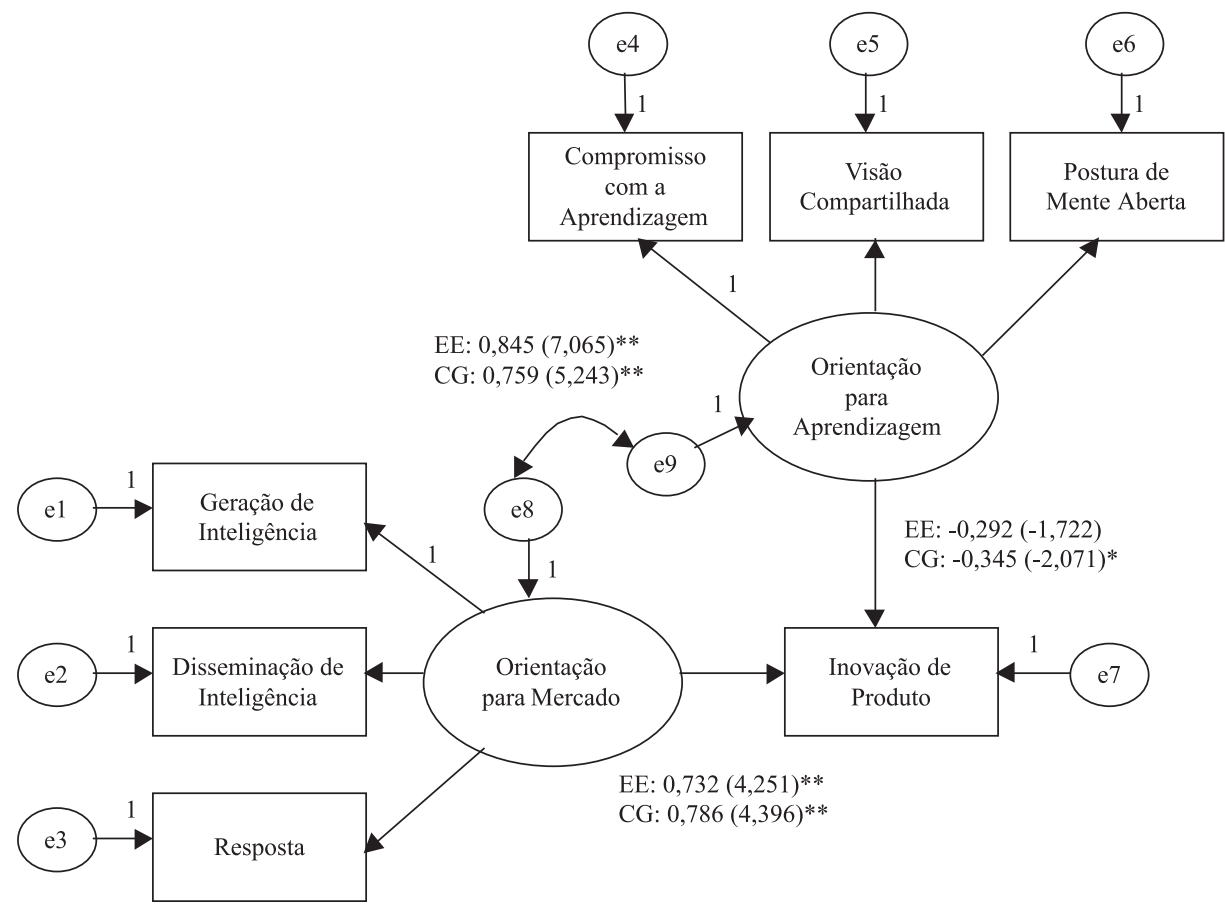

Notas: EE - loadings registrados na amostra da Indústria Eletro-Eletrônica. Os valores entre parênteses representam seus t-values correspondentes.

CG - loadings registrados na amostra dos Cursos de Graduação em Administração. Os valores entre parênteses representam os $t$-values correspondentes.

$* \mathrm{p}<0,05$

** $\mathrm{p}<0,01$

Para a simplificação do modelo, foram seguidas as recomendações de Bagozzi e Edwards (1998), quanto ao procedimento de agregação parcial do modelo de medidas. Esta simplificação é obtida pela substituição da variável latente no modelo por uma variável manifesta gerada a partir do cômputo da soma dos indicadores formadores da variável latente original, transformando construtos de primeira ordem em variáveis manifestas e, por conseqüência, os construtos de segunda ordem em construtos ou variáveis latentes de primeira ordem.

Destaca-se que o modelo de medidas simplificado permaneceu logicamente equivalente ao modelo teórico originalmente proposto. Em outras palavras, o modelo expresso na Figura 2 é equivalente ao modelo de Baker e Sinkula (1999a), apresentado na Figura 1, com a exceção da ausência do construto de desempenho organizacional, conforme já mencionado. 
A seguir são expostos os detalhes da análise dos resultados encontrados.

\section{Análise dos Resultados}

A análise da correlação entre os diversos indicadores mostrou que, nas duas amostras, existe correlação significativa $(p<0,01)$ entre os construtos inseridos no modelo. Estes resultados foram sintetizados por meio de uma análise de correlação, considerando as escalas agregadas (summated scales) (Hair et al., 1998), escalas estas representativas das dimensões de Orientação para o Mercado (OM) e Orientação para Aprendizagem (OA), além do construto de Inovação (IN). A Tabela 1 apresenta os coeficientes de correlação registrados.

\section{Tabela 1: Correlação entre as Dimensões de Orientação para o Mercado}

\begin{tabular}{c|cc|cc}
\hline & \multicolumn{2}{|c|}{ Eletroeletrônica } & \multicolumn{2}{c}{ Ensino } \\
\hline & OM & OA & OM & OA \\
\hline OA & $0,714^{* *}$ & & $0,643^{* *}$ & \\
IN & $0,444^{* *}$ & $0,296^{* *}$ & $0,479^{* *}$ & $0,232^{* *}$ \\
\hline
\end{tabular}

Fonte: coleta de dados

Nota: $* * \mathrm{p}<0,01$

Note-se que os valores são bastante semelhantes em ambas as amostras, denotando comportamento similar de associação entre os construtos para ambos os setores econômicos. Salienta-se, porém, o baixo coeficiente de correlação entre os construtos de Orientação para Aprendizagem e de Inovação, menores que 0,3 em ambos os casos. Ressalta-se também a forte associação entre os construtos de Orientação para o Mercado e de Orientação para Aprendizagem.

Estes resultados, em princípio, poderiam ser tomados como favoráveis a uma conclusão quanto à validade do modelo, a exemplo do estudo realizado por Faleiro (2001). Por outro lado, pode-se considerar também que os resultados apontam uma possível fragilidade do modelo em análise, sinalizando uma relação mais forte do construto de Inovação com o construto de Orientação para o Mercado do que com o construto de Orientação para Aprendizagem. Aparentemente, a Orientação para o Mercado e a Orientação para Aprendizagem se correlacionam fortemente, porém é a Orientação para o Mercado que mais influência exerce sobre a Inovação. Estas ponderações evidenciaram a necessidade de aplicação de uma técnica estatística mais acurada, como é o caso da modelagem de equações estruturais. 
Com este intuito, na análise fatorial confirmatória, examinaram-se inicialmente as propriedades relativas à validade dos construtos, quais sejam, a unidimensionalidade, a confiabilidade, a validade convergente e a validade discriminante, seguindo-se as recomendações de Garver e Mentzer (1999). Todos os construtos, em ambas as amostras, demonstraram validade de construto.

Quanto às medidas de ajustamento, os resultados apresentados em ambas as amostras foram muito similares, representando bons índices, conforme padrões supracitados sugeridos por Hair et al. (1998), e demonstrando a adequação do modelo teórico em ambas as amostras. A Tabela 2 relaciona os índices calculados.

\section{Tabela 2: Índices de Ajustamento}

\begin{tabular}{lcc}
\hline & Eletro & Ensino \\
\hline$\chi$ 2/GL & 2,09 & 1,38 \\
GFI & 0,962 & 0,962 \\
AGFI & 0,911 & 0,91 \\
TLI & 0,966 & 0,981 \\
CFI & 0,981 & 0,989 \\
RMSEA & 0,08 & 0,056 \\
\hline
\end{tabular}

Fonte: coleta de dados.

A partir da confirmação do ajustamento do modelo, foi possível a leitura dos parâmetros estimados para as relações de efeito direto hipotetizadas no modelo teórico. A Figura 2 apresenta os parâmetros em estudo. Note-se que a relação entre a Orientação para o Mercado e Inovação se mostrou positiva e significativa $(p<0,01)$ em ambas as amostras, registrando cargas fatoriais elevadas. Já a relação entre a Orientação para Aprendizagem e a Inovação não se mostrou significativa ( $>0,05)$ para a amostra da Indústria Eletroeletrônica, além de ter registrado cargas fatoriais negativas, ao contrário do que o modelo proposto hipotetizava.

Em outras palavras, a análise das cargas fatoriais aponta uma influência direta, forte e positiva da Orientação para o Mercado sobre a Inovação. Por outro lado, esta análise expõe uma influência não significativa da Orientação para Aprendizagem sobre a Inovação, na Indústria Eletroeletrônica, além da influência negativa da Orientação para Aprendizagem sobre a Inovação, no setor de Ensino. Ressalta-se que neste último caso, o nível de significância identificado para a relação esteve próximo do limite máximo aceitável $(\mathrm{p}=0,048)$.

Essas constatações diferem dos resultados encontrados por Baker e Sinkula (1999a). Tais autores encontraram relações positivas e significativas em ambas as relações, ou seja, da Orientação para Aprendizagem sobre a Inovação e 
da Orientação para o Mercado sobre a Inovação. Os resultados também contrastam com o diagnóstico elaborado por Claycomb e Germain (1997), para os quais a Orientação para Aprendizagem exerce influência positiva sobre a Inovação.

Uma possível explicação para estes resultados foi destacada por Perin (2002), para o qual a Orientação para Aprendizagem tem relação de efeito direto sobre a Orientação para o Mercado e não de associação, conforme definido no modelo proposto. Esta explicação encontra sustentação nas proposições teóricas de Day (1994a), Dickson (1996) e Hunt e Morgan (1996). Até mesmo, Baker e Sinkula (1999a) admitem explicitamente a existência desta corrente teórica alternativa à sua proposição. Neste sentido, uma modificação estrutural foi efetuada no modelo proposto, qual seja a substituição da relação de associação por uma relação de efeito direto da Orientação para Aprendizagem sobre a Orientação para o Mercado. Esta alteração permitiu a avaliação, por meio dos parâmetros estimados pela modelagem de equações estruturais, dos efeitos indiretos da Orientação para Aprendizagem sobre a Inovação, tendo a Orientação para o Mercado como variável mediadora.

A nova estimação de parâmetros, então, apresentou carga fatorial positiva e significativa $(p<0,01)$ na influência direta da Orientação para Aprendizagem sobre a Orientação para o Mercado, tanto na Indústria Eletroeletrônica (carga=0,832; $t$-value $=9,012$ ), quanto no setor de Ensino (carga=0,759; $t$-value=6,589). Os parâmetros relativos às duas outras relações permaneceram inalterados. Por sua vez, a relação de efeito indireto registrado entre Orientação para Aprendizagem e a Inovação de Produtos, mediante a Orientação para o Mercado, apresentouse significativa $(\mathrm{p}<0,01)$, sendo que na Indústria Eletroeletrônica o parâmetro estimado foi de 0,609, enquanto no setor de Ensino foi de 0,597.

Em síntese, estes resultados demonstram que há grande influência positiva e direta da Orientação para o Mercado sobre a Inovação de Produtos. O papel desempenhado pela Orientação para Aprendizagem é o de potenciar a Orientação para o Mercado e a relação desta com a Inovação de Produtos.

As considerações finais quanto a estes resultados são apresentadas a seguir.

\section{Conclusões}

As diversas correntes teóricas que tratam da Orientação para o Mercado e sua relação com o desempenho empresarial são unânimes em afirmar que este tema, 
apesar de bastante consolidado, ainda apresenta diversas facetas a serem desvendadas. Dentre estas facetas, destaca-se uma questão de pesquisa bastante freqüente nos estudos da área que diz respeito à insuficiência da postura de Orientação para o Mercado em propiciar às organizações uma vantagem competitiva de longo prazo. Uma série de estudos supracitados discute esta questão específica, manifestando a necessidade de uma ênfase da organização no processo de aprendizagem contínua, paralelamente à sua postura de Orientação para o Mercado, para a sustentação de uma posição distintiva e duradoura no ambiente competitivo. Neste sentido, este trabalho relacionou as proposições e conclusões dos principais trabalhos relativos à Orientação para o Mercado, Orientação para Aprendizagem e Inovação de Produtos.

O modelo teórico, inicialmente proposto, apresentou influência direta, forte e positiva da Orientação para o Mercado sobre a Inovação e influência não significativa e/ou negativa da Orientação para Aprendizagem sobre Inovação, resultados estes diferentes, parcialmente, daqueles encontrados nos estudos empreendidos por Baker e Sinkula (1999a) e Claycomb e Germain (1997). Em função desses achados, a estrutura do modelo inicial foi alterada e os resultados obtidos apontaram um efeito positivo e direto da Orientação para o Mercado sobre a Inovação e apenas um efeito indireto da Orientação para Aprendizagem sobre a Inovação. Ou seja, nos setores investigados é muito provável que o aumento no grau de Orientação para o Mercado explique o aumento do grau de Inovação, enquanto é pouco provável que o aumento do grau de Orientação para Aprendizagem explique o aumento do grau de Inovação, sem a correspondente elevação no grau de Orientação para o Mercado.

Essas constatações reforçam a indicação constante da literatura da área de Marketing de que uma postura de Orientação para o Mercado, associada a uma postura de Orientação para Aprendizagem, tem forte probabilidade de influenciar positivamente a Inovação de Produtos nas organizações, contribuindo para a obtenção de vantagem competitiva a longo prazo (Slater e Narver, 1995; Dickson, 1996; Hurley e Hult, 1998; Baker e Sinkula, 1999b).

Para tanto, as recomendações de Day (1994a), quanto à aprendizagem contínua sobre o mercado, se aplicam com muita propriedade. Este autor sugere a captação de informações com uma visão periférica, objetivando a identificação de oportunidades que extrapolem o modelo mental normalmente utilizado para analisar o mercado. Com isso, os gerentes podem sobrepor o conjunto de informações provenientes das fontes mais freqüentes ou usuais, as quais tendem a sustentar o modelo mental vigente. 
Os resultados sugerem que as organizações devam incrementar a geração e a disseminação de informações de mercado por meio da organização para promover respostas mais efetivas às oportunidades e ameaças identificadas no mercado. Esta postura influenciará positivamente a Inovação de Produtos.

O estudo demonstrou que a Orientação para Aprendizagem pode potenciar significativamente a Orientação para o Mercado, afetando indiretamente e de forma positiva a Inovação. Neste sentido, parece ser razoável o argumento de que uma organização deveria estimular em seus funcionários a postura de compromisso com a aprendizagem e de mente aberta, valorizando a iniciativa de rompimento dos paradigmas vigentes na organização. Seria razoável também o compartilhamento da visão de futuro idealizada para a organização com os funcionários dos seus diversos níveis.

\section{Limitações da Pesquisa e Sugestões para Estudos Futuros}

Apesar do rigor do método aplicado nessa pesquisa, algumas situações vivenciadas durante o desenvolvimento do trabalho sugerem certas limitações, e a superação de cada uma dessas limitações resultará em recomendações para pesquisas futuras.

Os levantamentos executados foram do tipo corte transversal (cross-sectional), cuja mensuração das variáveis ocorreram em momentos similares, impedindo, portanto, analisar modificações que pudessem ocorrer em diferentes momentos de tempo, o que possibilitaria verificar associações e comparar com os resultados deste estudo.

A percepção de um único respondente por unidade amostral pode distorcer as verdadeiras práticas adotadas na organização. Desta forma, seria importante que estudos futuros considerassem outras possibilidades, tais como respondentes múltiplos, respondentes de outros escalões da organização ou ainda respondentes externos à organização.

Os dados foram coletados com base nas percepções, opiniões e avaliações subjetivas dos respondentes. Especialmente em relação ao construto de Inovação, estudos futuros poderiam empregar medidas objetivas para tal mensuração.

Por fim, apesar da preocupação com a análise do viés dos não respondentes, salienta-se que essa pesquisa utilizou amostras não probabilísticas de retorno de correio. Seria interessante que trabalhos futuros utilizassem mais de um método de coleta de dados, observando a aleatoriedade na seleção das unidades amostrais. 


\title{
Notas
}

\begin{abstract}
${ }^{1}$ Parte deste estudo foi financiado pela Fundação de Amparo à Pesquisa do Estado do Rio Grande do Sul (FAPERGS).

${ }^{2}$ Chou e Bentler (1995) afirmam que os dados reais coletados em pesquisas sociais quase nunca apresentam distribuição normal e, em função deste fato, os pesquisadores têm-se preocupado particularmente com a seleção de métodos de estimação adequados para variáveis com distribuição não normal.

${ }^{3}$ Hair et al. (1998) definem bootstrapping como um processo de estimação por meio do qual os erros padronizados não são calculados com a concepção estatística, mas sim baseados nas observações empíricas (pela replicação dos dados originais da amostra).
\end{abstract}

\section{Bibliografia}

BAGOZZI, R. P.; EDWARDS, J. R.

A general approach for representing constructs in organizational research.

\section{Organizational Research}

Methods, v. 1, n. 1, p. 45-87, Jan. 1998.

BAKER, W. E.;

SINKULA, J. M.

The synergetic effect of market orientation and learning orientation on organizational performance. Journal of the Academy of Marketing Science, v. 27, n. 4, p. 411-427, Fall 1999a.

Orientation, market orientation, and innovation: integrating and extending models of organizational performance. Journal of $M$ a r k e t - F o c u s e d Management, v. 4, n. 4, p. 295308, Dec. 1999b.
BECKER, J.;

HOMBURG, C.

Market-oriented management: a systems-based perspective. Journal of Market-Focused Management, v. 4, n. 1, p.17-41, June 1999.

CARUANA, A.;

RAMASESHAN, B.; EWING, M.

Market orientation and performance: a study of Australasian universities. Curtin Business School, Nov. 1996. Working Paper Series.

\section{CHOU, C.-P.;}

BENTLER, P. M.

Estimates and tests in structural equation modeling. In: HOYLE, R. $H$ (Ed.). Structural equation modeling: concepts, issues, and applications. London: Sage Publications, 1995. 
CHURCHILL JR., G. A.

Marketing research:

methodological foundations.

Orlando: The Dryden Press, 1999.

CLAYCOMB, C.;

GERMAIN, R.

Organizational learning and performance: an empirical test. In: AMERICAN MARKETING ASSOCIATION WINTER EDUCATORS' CONFERENCE, 1997, Saint Petersburg. Proceedings... Saint Petersburg: AMA, 1997. p. 94-100.

DAMANPOUR, F.

Organizational innovation: a metaanalysis of effects of determinants and moderators. Academy of Management Journal, v. 34, n. 3, p. 555-590, 1991.

DAY, G. S.

Continuous learning about markets. California Management Review, v. 36, n. 4, p 9-31, Summer 1994a.

The capabilities of market-driven organizations. Journal of Marketing, v. 58, n. 4, p 37-52, Oct. 1994b.

DESHPANDÉ, R.;

FARLEY, J. U.

Measuring market orientation: generalization and synthesis. Journal of Market Focused Management, v. 2, n. 3, p. 213232, 1998.
DESHPANDÉ, R.;

FARLEY, J. U.;

WEBSTER JR., F. E.

Corporate culture, customer orientation, and innovativeness in Japanese firms: a quadrad analysis. Journal of Marketing, v. 57, n. 1, p. 23-27, Jan. 1993.

DICKSON, P. R.

The static and dynamic mechanics of competition: a comment on Hunt and Morgan's comparative advantage theory. Journal of Marketing, v. 60, n. 3, p. 102-106, 1996.

DRUCKER, P. F.

The practice of management.

New York: Harper \& Row, 1954.

EGEREN, M. V.;

O'CONNOR, S.

Drivers of market orientation and performance in service firms. Journal of Services Marketing, v. 12, n. 1, p. 39-58, 1998.

FALEIRO, S. N.

A relação entre orientação para o mercado, orientação para a aprendizagem e inovação: o caso dos cursos de graduação em administração filiados à ANGRAD. Porto Alegre, 2001. Dissertação (Mestrado em Administração) Programa de Pós-Graduação em Administração, Universidade Federal do Rio Grande do Sul. 
FARRELL, M. A.

Developing a market-oriented learning organisation. Australian Journal of Management, v. 25, n. 2, p. 201-222, Sept. 2000.

FRITZ, W..

Market orientation and corporate success: findings from Germany. European Journal of Marketing, v. 30, n. 8, p. 59-74, 1996.

GALBRAITH, J. R.

Projetando a organização inovadora. In: STARKEY, K. (Org). Como as organizações aprendem. São Paulo: Futura, 1997. p.190-218.

GARVER, M. S.;

MENTZER, J. T.

Logistics research methods: employing structural equation modeling to test for construct validity. Journal of Business Logistics, v. 20, n. 1, p. 33-57, 1999.

HAIR JR., J. F. et al.

Multivariate data analysis. 5.ed. Englewood Cliffs, NJ: Prentice Hall, 1998.

HAN, J. K.;

KIM, N.;

SRIVASTAVA, R. K.

Market orientation and organizational performance: is innovation a missing link? Journal of Marketing, v. 62, n. 4, p. 3045, Oct. 1998.
HENARD, D. H.;

SZYMANSKI, D. M.

Why some new products are more successful than others. Journal of Marketing Research, v. 38, p. 362-375, 2001.

HOYLE, R. H. (Ed.).

Structural equation modeling: concepts, issues, and applications. London: Sage Publications, 1995.

HUNT, S. D.;

MORGAN, R. M.

The resource-advantage theory of competition: dynamics, path dependencies, and evolutionary dimensions. Journal of Marketing, v. 60, n. 2, p. 107-114, 1996.

HURLEY, R. F.;

HULT, G. T. M.

Innovation, market orientation, and organizational learning: an integration and empirical examination. Journal of Marketing, v. 62, n. 3, p. 42-54, July 1998.

JAWORSKI, B. J.;

KOHLI, A. K.

Market orientation: antecedents and consequences. Journal of Marketing, v. 57, p. 53-71, July 1993. 
KIMBERLY, J. R.

Managerial innovation. In: NYSTROM, P. C.; STARBUCK, W. H. (Eds.). Handbook of organizational design: adapting organizations to their environments. New York: Oxford University Press, 1981.

KOHLI, A. K.;

JAWORSKI, B. J.

Market orientation: the construct, research propositions, and managerial implications. Journal of Marketing, v. 54, n. 2, p. 1-18, Apr. 1990.

KOHLI, A. K.;

JAWORSKI, B. J.;

KUMAR, A. MARKOR:

a measure of market orientation. Journal of Marketing Research, v. 30, n. 4, p. 467-477, Nov. 1993.

LUKAS, B. A.; FERRELL, O. C.

The effect of market orientation on product innovation. Journal of the Academy of Marketing Science, v. 28, n. 2, p. 239-247, Spring 2000.

MALHOTRA, N. K.

Pesquisa de marketing: uma orientação aplicada. Porto Alegre: Bookman, 2001.
MONTOYA-WEISS, M. M.;

CALANTONE, R.

Determinants of new product performance: a review and metaanalysis. Journal of Product Innovation Management, v. 11, p. 397-417, 1994.

NARVER, J. C.;

SLATER, S. F.

The effect of market orientation on business profitability. Journal of Marketing, v. 54, n. 4, p. 2035, Oct. 1990.

NARVER, J.C.;

SLATER, S. F.;

TIETJE, B.

Creating a market orientation. Journal of Market-Focused Management, v. 2, n. 3, p. 241255, 1998.

NEVIS, E. C.;

DIBELLA, A. J.;

GOULD, J. M.

Understanding organizations as learning systems. Sloan Management Review, v. 36, n. 2, p. 73-85, Winter 1995.

PELHAM, A. M.

Mediating influences on the relationship between market orientation and profitability in small industrial firms. Journal of Marketing Theory and Practice, v. 5, n. 3, p. 55-76, Summer 1997. 
PERIN, M. G.

A relação entre orientação para o mercado, aprendizagem organizacional e performance. Porto Alegre, 2002. Tese (Doutorado em Administração) Programa de Pós-Graduação em Administração, Universidade Federal do Rio Grande do Sul.

\section{PITT, L.;}

CARUANA, A.;

BERTHON, P. R.

Market orientation and business performance: some European evidence.

International

Marketing Review, v. 13, n. 1, p. 5-18, 1996.

PROBST, G.;

BUCHEL, B. S. T.

Organizational learning. Englewood Cliffs, NJ: Prentice Hall, 1997.

SAMPAIO, C. H.

Relação entre orientação para o mercado e performance empresarial em empresas de varejo de vestuário do Brasil. Porto Alegre, 2000. Tese (Doutorado em Administração) Programa de Pós-Graduação em Administração, Universidade Federal do Rio Grande do Sul.

SIGUAW, J. A.;

SIMPSON, P. M.;

BAKER, T. L.

Effects of supplier market orientation on distributor market orientation and the channel relationship: the distributor perspective. Journal of Marketing, v. 62, n. 3, p. 99-111, July 1998.

SINKULA, J. M.

Market information processing and organizational learning. Journal of Marketing, v. 58, n. 1, p. 35-45, Jan. 1994.

SINKULA, J. M.;

BAKER, W. E.; NOORDEWIER, T.

A framework for market-based organizational learning: linking values, knowledge and behavior. Journal of the Academy of Marketing Science, v. 25, n. 4, p. 305-318, 1997.

SLATER, S. F.;

NARVER, J. C.

Intelligence generation and superior customer value. Journal of the Academy of Marketing Science, v. 28, n. 1, p. 120-127, 2000.

Market orientation and the learning organization. Journal of Marketing, v. 59, n. 3, p. 63-74, July 1995.

Does competitive environment moderate the market orientation performance relationship? Journal of Marketing, v. 58, n. 1, p. 46-55, Jan. 1994. 
TUSHMAN, M.;

NADLER, D.

Organizando-se para a inovação. In: STARKEY, K. (Org.). Como as organizações aprendem. São Paulo: Futura, 1997. p. 166-189.

ULLMAN, J. M.

Structural equation modeling. In: TABACHNICK, B. G.; FIDELL, L. S. Using multivariate statistics. Boston: Allyn \& Bacon, 2000.
UNCLES, M.

Market orientation. Australian Journal of Management, v. 25, n. 2, p. i-ix, Sept. 2000.

WEST, S. G.;

FINCH, J. F.;

CURRAN, P. J.

Structural equation models with nonnormal variables: problems and remedies. In: HOYLE, R. H. (Ed.). Structural equation modeling: concepts, issues, and applications. London: Sage Publications, 1995. 